Isabela Franco Cavalcanti ${ }^{1}$

Polyana da Nóbrega Farias ${ }^{2}$

LUCAS ITHAMAR ${ }^{3}$

VILMA MARIA DA SILVA ${ }^{4}$

ANDREA LEMOS 3

Artigo Original

Palavras-chave

Saúde da mulher

Climatério/metabolismo

Disfunção sexual fisiológica

Sexualidade

Keywords

Women's health

Climacteric/metabolism

Sexual dysfunction, physiologic

Sexuality

\title{
Função sexual e fatores associados à disfunção sexual em mulheres no climatério
}

\author{
Sexual function and factors associated with sexual dysunction \\ in climacteric women
}

\section{Resumo}

OBJETIVO: Avaliar a função sexual e os fatores associados à disfunção sexual de mulheres no período do climatério. MÉTODOS: Foi realizado um estudo de corte transversal incluindo 173 mulheres, na faixa etária de 35 a 65 anos, com parceiro fixo nos últimos 6 meses, alfabetizadas, sem comprometimento cognitivo e com atividade sexual há pelo menos 6 meses. $\bigcirc$ instrumento utilizado para avaliar o desempenho sexual foi o Quociente Sexual, versão feminina. A associação entre disfunção sexual e dados sociodemográficos, antecedentes pessoais e obstétricos e história sexual foi realizada por meio do teste do $\chi^{2}$ de Pearson e da força de associação, por meio do odds ratio (OR) com intervalo de confiança de 95\% (IC95\%). RESULTADOS: Neste estudo, 46,2\% das mulheres apresentaram disfunção sexual. Houve uma diminuição da chance de disfunção sexual para a faixa etária entre 35 e 49 anos (OR=0,3; IC95\% $0,2-0,6)$ e para as mulheres que se sentiam à vontade para falar sobre sexo $(O R=0,5 ; I C 95 \% 0,2-0,8)$. Entretanto, presença de osteoporose $(O R=3,3$; IC95\% 1,5-7,6), incontinência urinária $(O R=2,0 ; I C 95 \% 1,1-3,7)$ e correções cirúrgicas do assoalho pélvico $(O R=2,2 ; \mathrm{IC} 95 \% 1,1-4,5)$ elevaram essa chance. CONCLUSÕES: A frequência de disfunção sexual em mulheres na faixa etária entre 35 e 65 anos foi 46,2\% e fatores como osteoporose, incontinência urinária e correções cirúrgicas do assoalho pélvico aumentaram a chance de disfunção sexual.

\section{Abstract}

PURPOSE: To evaluate the sexual function and factors associated with sexual dysfunction in climacteric women. METHODS: A cross-sectional study was conducted on 173 women aged 35 to 65 years old, with a steady partner during the last 6 months, who are literate, without cognitive impairment, and with sexual activity for at least 6 months. The instrument used to assess sexual performance was the Sexual Quotient, female version. The association between sexual dysfunction and sociodemographic data, personal, obstetric and sexual history was determined by Pearson's $\chi^{2}$ test and strength of association by the odds ratio (OR) with a $95 \%$ confidence interval $(95 \% \mathrm{Cl})$. RESULTS: In this study, $46.2 \%$ of the women reported sexual dysfunction. There was a decrease in the chance of sexual dysfunction for the age group between 35 and 49 years old $(O R=0.3 ; 95 \% \mathrm{Cl} 0.2-0.6)$ and for women who felt comfortable talking about $\operatorname{sex}(\mathrm{OR}=0.5 ; 95 \% \mathrm{Cl} 0.2-0.8)$. However, the presence of osteoporosis $(\mathrm{OR}=3.3 ; 95 \% \mathrm{Cl} 1.5-7.6)$, urinary incontinence $(O R=2.0 ; 95 \% \mathrm{Cl} 1.1-3.7)$, and surgical corrections of the pelvic floor $(O R=2.2 ; 95 \% \mathrm{Cl} 1.1-4.5)$ increased this chance. CONCLUSIONS: The frequency of sexual dysfunction in women aged 35 to 65 years old was $46.2 \%$ and factors such as osteoporosis, urinary incontinence and surgical corrections of the pelvic floor increased the chance of sexual dysfunction.

Correspondência

Isabela Franco Cavalcanti Avenida Professor Moraes Rego, 1.235 - Cidade Universitárii (EP: $50670-901$

Recife (PE), Brasil

Recebido $13 / 03 / 2014$

Aceito com modificacōos $23 / 09 / 2014$
Ambulatório de Climatério do Hospital das Clínicas da Universidade Federal de Pernambuco - UFPE - Recife (PE), Brasil. IPrograma de Residência Multiprofissional Integrada em Saúde do Hospital das Clínicas, Universidade Federal de Pernambuco - UFPE Recife (PE), Brasil.

2Programa de Pós-Graduação do Departamento de Fisioterapia, Universidade Federal de Pernambuco - UFPE - Recife (PE), Brasil. ${ }^{3}$ Departamento de Fisioterapia, Universidade Federal de Pernambuco - UFPE - Recife (PE), Brasil.

${ }^{4}$ Serviço de Ginecologia e Obstetrícia do Hospital das Clínicas, Universidade Federal de Pernambuco - UFPE - Recife (PE), Brasil. Conflito de interesses: não há. 
Introdução

As queixas sexuais podem ocorrer durante toda a vida reprodutiva, mas na fase do climatério as mulheres tornam-se mais vulneráveis à disfunção sexual em consequência da interação de um conjunto complexo de fatores, tais como o hipoestrogenismo fisiológico e as dificuldades nos aspectos emocional e social característicos dessa fase ${ }^{1-3}$. Além desses fatores, a função sexual prévia da mulher, na idade reprodutiva, também é considerada um fator crucial para a função sexual no climatério ${ }^{4}$.

No Brasil, a prevalência de disfunção sexual chega a atingir $49 \%$ das mulheres com 18 anos ou mais ${ }^{5}$ e $67 \%$ daquelas na meia-idade (40 a 65 anos $)^{6}$. Além disso, 60\% das brasileiras referem ter diminuição da atividade sexual após a menopausa ${ }^{7}$.

Atualmente, existem no Brasil cerca de 30 milhões de mulheres entre 35 e 65 anos, o que significa que $32 \%$ da população feminina estão na faixa etária em que ocorre o climatério e, com o aumento da expectativa de vida mundial, essa porcentagem tende a aumentar ${ }^{8}$. Como a sexualidade é um dos pilares para o envelhecimento sadio e com qualidade de vida, é fundamental o conhecimento dos fatores que podem interferir na vida sexual durante essa fase.

No entanto, são escassos os estudos que avaliam a sexualidade das brasileiras no climatério ${ }^{2,6,7,9}$. Isso se deve, provavelmente, às questões culturais envolvendo o tema. Embora exista uma maior quantidade de estudos internacionais quanto à sexualidade de mulheres no climatério, não há consenso na literatura sobre os fatores que influenciam a vida sexual nessa fase da vida da mulher ${ }^{6,10-12}$.

Baseado no exposto, esta pesquisa buscou avaliar a função sexual e os fatores associados à disfunção sexual em mulheres atendidas no ambulatório de climatério de um hospital-escola em Pernambuco, Brasil.

\section{Métodos}

Trata-se de um estudo de corte transversal realizado em um hospital-escola no Recife, Pernambuco, Brasil. A amostra foi calculada por meio do programa Epi-Info, versão 3.4.3- 2007. Utilizou-se a frequência do evento (disfunção sexual) de $49 \%$, com erro absoluto de $10 \%$ e nível de confiança de $99 \%$, totalizando um número de 166 mulheres. Adicionou-se 5\% de perdas, o que resultou em uma amostra final de 173 mulheres.

As voluntárias foram recrutadas de forma sequencial, por conveniência, enquanto aguardavam a consulta no ambulatório de climatério, de maio de 2012 a novembro de 2013. Antes da inclusão, elas foram esclarecidas quanto aos objetivos, riscos e benefícios da pesquisa e aquelas que desejaram participar assinaram o Termo de Consentimento Livre e Esclarecido (TCLE).
Foram considerados como critérios de inclusão mulheres com idades entre 35 e 65 anos e com parceiro fixo nos últimos 6 meses, e de exclusão, comprometimento cognitivo que impossibilitasse a compreensão das questões, analfabetismo e abstinência sexual nos últimos 6 meses.

Para a coleta dos dados, foram utilizados dois instrumentos: o primeiro foi um formulário desenvolvido especificamente para esta pesquisa, no qual constavam dados sociodemográficos (idade, ocupação, estado civil, religião e escolaridade); antecedentes pessoais (comorbidades, etilismo, tabagismo, terapia de reposição hormonal e cirurgias ginecológicas); antecedentes obstétricos (parto vaginal, história do uso de fórceps e de episiotomia) e história sexual (coitarca, sentir-se à vontade para falar sobre sexo, masturbação, uso de preservativo e satisfação com a vida sexual). O segundo instrumento foi o Quociente Sexual, versão feminina (QS-F) .

O QS-F é um questionário composto por dez perguntas autorresponsivas, desenvolvido e validado especificamente para a população feminina brasileira, pelo Programa de Estudos em Sexualidade do Instituto de Psiquiatria do Hospital das Clínicas da Faculdade de Medicina da Universidade de São Paulo. Ele avalia as diversas fases do ciclo da resposta sexual, além de outros domínios: desejo e interesse sexual, preliminares, excitação pessoal e sintonia com o parceiro, conforto, orgasmo e satisfação, permitindo identificar disfunções específicas e dificuldades sexuais ${ }^{7}$.

Cada questão do QS-F é respondida em uma escala gradual de zero a cinco, com zero indicando "nunca" e cinco, "sempre". O escore é obtido pela soma dos pontos de todas as questões multiplicada por 2 , resultando em uma pontuação que varia entre 0 e 100 , com valores maiores informando melhor desempenho/satisfação sexual. Entretanto, a questão sete deve ser transformada antes desse cálculo, por estar no sentido reverso, obedecendo à seguinte forma: Q7 rever$\mathrm{sa}=5-\mathrm{Q} 7$, conforme indicado por Abdo et al. ${ }^{5}$. É estabelecido um escore menor ou igual a 60 como ponto de corte para diagnosticar a presença de disfunção sexual femininas.

A análise dos dados foi realizada com o uso do software Statistical Package for Social Sciences (SPSS), versão 18 para Windows. Para a caracterização da amostra, calcularam-se a média, o desvio padrão (DP) e o intervalo de confiança para as variáveis idade e escore do QS-F. Foi aplicado o teste Kolmogorov-Smirnov a fim de verificar se as variáveis contínuas apresentavam distribuição normal. Na análise descritiva, os dados foram expostos em tabelas de distribuição das frequências absoluta e relativa. Para verificar a presença de associação entre a variável dependente do estudo (disfunção sexual) e as independentes (dados sociodemográficos, antecedentes pessoais e obstétricos e história sexual), foi utilizado o $\chi^{2}$ de Pearson. Para analisar a força da associação, utilizou-se o odds ratio (OR) com intervalo de confiança de 95\% (IC95\%). 
Esta investigação foi desenvolvida conforme as recomendações da Resolução n ${ }^{\circ}$ 466/2012 do Conselho Nacional de Saúde do Ministério da Saúde ${ }^{13}$, que dispõe sobre as pesquisas envolvendo seres humanos, após aprovação pelo Comitê de Ética em Pesquisa do Centro de Ciências da Saúde da Universidade Federal de Pernambuco, sob $n^{\circ}$ de CAAE 00714912.8.0000.5208.

\section{Resultados}

Das 338 usuárias abordadas, 5 não aceitaram participar da pesquisa, permanecendo 333 voluntárias. Dessas, 109 foram inelegíveis (faixa etária diferente de 35 a 65 anos ou ausência de parceiro fixo nos últimos 6 meses) e 51 foram elegíveis, mas não recrutadas (comprometimento cognitivo, analfabetismo ou abstinência sexual nos últimos 6 meses), restando somente 173 mulheres para o estudo.

A média de idade das 173 voluntárias avaliadas foi de 49,9 anos (DP=8,5; IC95\% 48,6-51,1) e do escore do QS-F foi de 61,6 pontos (DP=23,0; IC95\% 58,1-65,0).

A maioria das mulheres incluídas era dona de casa (60,7\% contra 39,3\% que tinham trabalho assalariado); casada ou vivia em unãa estável $(85,5 \%$ contra $14,5 \%$ solteiras ou com outro estado civil); católica ( $52,6 \%$ contra $41 \%$ evangélicas, $3,5 \%$ espíritas e $2,9 \%$ pertencentes a outras religiões) e tinha até 9 anos de estudo $(67,6 \%$ contra $32,4 \%$ com 10 ou mais anos de estudo).

Do total, 73,9\% tinham pelo menos uma comorbidade, sendo a hipertensão arterial $(43,9 \%)$, a incontinência urinária $(41 \%)$ e a osteoporose (19\%) as de maiores prevalências.

Das 128 voluntárias que afirmaram terem comorbidades, 31,2\% alegaram que essas doenças influenciavam negativamente suas vidas sexuais.

Quanto aos hábitos de vida, nenhuma das mulheres referiu fazer uso diário de álcool e $9(5,2 \%)$ relataram serem tabagistas. No que se refere à história sexual, a média de idade da coitarca foi de 19,3 anos ( $\mathrm{DP}=4,4), 13,8 \%$ das voluntárias mencionaram masturbar-se e $68,7 \%$ alegaram nunca terem usado preservativo, seja feminino ou masculino, nas relações sexuais. Além disso, 34,6\% das participantes relataram estarem insatisfeitas com a vida sexual.

Da amostra total, 80 mulheres $(46,2 \%)$ apresentaram disfunção sexual segundo o escore do QS-F. Ainda de acordo com o escore desse quociente, $4 \%$ delas exibiram padrão de desempenho sexual de nulo a ruim, $17,9 \%$ de ruim a desfavorável, $24,3 \%$ de desfavorável a regular, $30,1 \%$ de regular a bom e $23,7 \%$ de bom à excelente.

Ao dicotomizar a amostra em dois subgrupos quanto à presença ou ausência de disfunção sexual, baseada nos escores do QS-F, foram realizadas associações com as variáveis independentes. Em relação aos dados sociodemográficos, houve diminuição da chance de disfunção sexual para a faixa etária entre 35 e 49 anos $(\mathrm{OR}=0,3$;
IC95\% 0,2-0,6), porém não houve associação do estado civil e da escolaridade à disfunção sexual (Tabela 1).

Quanto aos antecedentes pessoais, houve associação da presença de osteoporose $(\mathrm{OR}=3,3$; IC95\% 1,5-7,6), incontinência urinária $(\mathrm{OR}=2,0$; IC95\% 1,1-3,7) e correções cirúrgicas do assoalho pélvico $(\mathrm{OR}=2,2$; IC95\% $1,1-4,5)$ à disfunção sexual. Hipertensão arterial, depressão, diabetes melito, influência das comorbidades na função sexual, uso de terapia de reposição hormonal por seis ou mais meses, histerectomia e ooforectomia não apresentaram tal associação (Tabela 2).

Em relação aos antecedentes obstétricos, não houve associação entre as variáveis parto vaginal, uso de fórceps, episiotomia e disfunção sexual (Tabela 3).

Tabela 1. Associação entre a presença da disfunção sexual, mensurada pelo escore do Quociente Sexual Feminino, e os dados sociodemográficos de mulheres no climatério na faixa etária entre 35 e 65 anos

\begin{tabular}{|c|c|c|c|c|c|}
\hline \multirow[b]{2}{*}{ Dados sociodemográficos } & \multicolumn{2}{|c|}{ QS-F } & \multirow[b]{2}{*}{$\begin{array}{c}\text { Valor } \\
\mathbf{p}^{*}\end{array}$} & \multirow[b]{2}{*}{ OR } & \multirow[b]{2}{*}{ IC95\% } \\
\hline & $\begin{array}{c}\text { CDS } \\
\mathrm{n}(\%)\end{array}$ & $\begin{array}{c}\text { SDS } \\
n(\%)\end{array}$ & & & \\
\hline \multicolumn{6}{|l|}{ Faixa etária } \\
\hline 35 a 49 anos & $28(32,9)$ & $57(67,1)$ & $0,001^{\star \star}$ & 0,3 & $0,2-0,6$ \\
\hline 50 a 65 anos & $52(59,1)$ & $36(40,9)$ & & & \\
\hline \multicolumn{6}{|l|}{ Estado civil } \\
\hline Casada ov em união estável & $72(48,6)$ & $76(51,4)$ & 0,1 & 2,0 & $0,8-4,9$ \\
\hline Solteira ou outro & $8(32,0)$ & $17(68,0)$ & & & \\
\hline \multicolumn{6}{|l|}{ Escolaridade } \\
\hline Até 9 anos de estudo & $57(48,7)$ & $60(51,3)$ & 0,3 & 1,4 & $0,7-2,6$ \\
\hline$\geq 10$ anos de estudo & $23(41,1)$ & $33(58,9)$ & & & \\
\hline
\end{tabular}

QSF: Quociente Sexual Feminino; CDS: com disfunção sexual; SDS: sem disfunção sexual; ${ }^{*} \chi^{2}$ de Pearson; ${ }^{*}$ p $<0,05$; OR: odds ratio; IC95\%: intervalo de confiança de $95 \%$.

Tabela 2. Associação entre a presença da disfunç̃o sexual, mensurada pelo escore do Quociente Sexual Feminino, e os antecedentes pessoais de mulheres no climatério na faixa etária entre 35 e 65 anos

\begin{tabular}{|c|c|c|c|c|c|}
\hline \multirow[b]{2}{*}{ Antecedentes pessoais } & \multicolumn{2}{|c|}{ QS-F } & \multirow{2}{*}{$\begin{array}{c}\text { Valor } \\
\text { p* }^{*}\end{array}$} & \multirow[b]{2}{*}{ OR } & \multirow[b]{2}{*}{ IC95\% } \\
\hline & $\begin{array}{l}\text { CDS } \\
\text { n (\%) }\end{array}$ & $\begin{array}{c}\text { SDS } \\
\text { n (\%) }\end{array}$ & & & \\
\hline Hipertensão arterial & $41(53,9)$ & $35(46,1)$ & 0,07 & 1,7 & $0,9-3,2$ \\
\hline Depressã̃o & $13(52,0)$ & $12(48,2)$ & 0,5 & 1,3 & $0,6-3,1$ \\
\hline Diabetes melito & $11(45,8)$ & $13(54,2)$ & 1,0 & 1,0 & $0,4-2,3$ \\
\hline Osteoporose & $23(69,7)$ & $10(30,3)$ & $0,003^{\star \star}$ & 3,3 & $1,5-7,6$ \\
\hline Incontinência urinária & $40(56,3)$ & $31(43,7)$ & $0,03^{\star \star}$ & 2,0 & $1,1-3,7$ \\
\hline $\begin{array}{l}\text { Influência das comorbidades } \\
\text { na função sexual }{ }^{\#}\end{array}$ & $25(62,5)$ & $15(37,5)$ & 0,1 & 1,8 & $0,8-3,9$ \\
\hline TRH ( $\geq 6$ meses) & $10(47,6)$ & $11(52,4)$ & 0,9 & 1,1 & $0,4-2,6$ \\
\hline Histerectomia & $26(49,1)$ & $27(50,9)$ & 0,6 & 1,2 & $0,6-2,2$ \\
\hline Ooforectomia & $18(60,0)$ & $12(40,0)$ & 0,1 & 1,9 & $0,9-4,4$ \\
\hline $\begin{array}{l}\text { Correç̃̃es cirúrgicas do } \\
\text { assoalho pélvico }\end{array}$ & $25(61,0)$ & $16(39,0)$ & $0,03^{\star \star}$ & 2,2 & $1,1-4,5$ \\
\hline
\end{tabular}

QS-F: Quociente Sexual Feminino; CDS: com disfunção sexual; SDS: sem disfunção sexual; ${ }^{*} \chi^{2}$ de Pearson; ${ }^{*} p<0,05$; OR: odds ratio; IC95\%: intervalo de confiança de $95 \%$; ${ }^{n}=128$, referente às voluntárias com comorbidades; TRH: terapia de reposição hormonal. 
Tabela 3. Associação entre a presença da disfunção sexual, mensurada pelo escore do Quociente Sexual Feminino, e os antecedentes obstétricos de mulheres no climatério na faixa etária entre 35 e 65 anos

\begin{tabular}{|c|c|c|c|c|c|}
\hline \multirow[b]{2}{*}{ Antecedentes obstétricos } & \multicolumn{2}{|c|}{ QS-F } & \multirow{2}{*}{$\begin{array}{c}\text { Valor } \\
\mathbf{p}^{*}\end{array}$} & \multirow[b]{2}{*}{ OR } & \multirow[b]{2}{*}{ IC95\% } \\
\hline & $\begin{array}{c}\text { CDS } \\
\mathbf{n}(\%)\end{array}$ & $\begin{array}{c}\text { SDS } \\
\mathrm{n}(\%)\end{array}$ & & & \\
\hline \multicolumn{6}{|l|}{ Parto vaginal } \\
\hline Nenhum & $22(47,8)$ & $24(52,2)$ & 0,8 & 1,1 & $0,5-2,1$ \\
\hline Um ou mais partos & $58(45,7)$ & $69(54,3)$ & & & \\
\hline \multicolumn{6}{|l|}{ História de uso de fórceps } \\
\hline Sim & $11(61,1)$ & $7(38,9)$ & 0,2 & 2,0 & $0,7-5,5$ \\
\hline Não & $69(44,5)$ & $89(55,5)$ & & & \\
\hline \multicolumn{6}{|l|}{ História de episiotomia } \\
\hline $\operatorname{Sim}$ & $50(45,9)$ & $59(54,1)$ & 0,9 & 1,0 & $0,5-1,8$ \\
\hline Não & $30(46,9)$ & $34(53,1)$ & & & \\
\hline
\end{tabular}

QS-F: Quociente Sexual Feminino; CDS: com disfunção sexual; SDS: sem disfunção sexual; * $\chi^{2}$ de Pearson; OR: odds ratio; IC95\%: intervalo de confiança de $95 \%$.

Quanto à história sexual, sentir-se à vontade para falar sobre sexo esteve presente em $33(37,1 \%)$ mulheres com disfunção sexual e em $56(62,9 \%)$ sem $(p=0,01)$, e houve diminuição da chance de disfunção sexual para as mulheres que apresentavam este comportamento $(\mathrm{OR}=0,5 ; \mathrm{IC} 95 \% 0,2-0,8)$.

\section{Discussão}

No presente estudo, 46,2\% das mulheres no climatério apresentaram disfunção sexual. Esse valor foi bem próximo aos $49 \%$ encontrados em um estudo nacional, com uma amostra de 1.219 mulheres de 18 anos ou mais 5 . No entanto, foi inferior aos $67 \%$ detectados em outra investigação realizada no Brasil com 370 mulheres de 40 a 65 anos, faixa etária bastante semelhante à desta pesquisa, mas que utilizou um instrumento de coleta distinto, o Female Sexual Function Index (FSFI) ${ }^{2}$, o que pode ter contribuído para essa diferença nos resultados.

Um dado interessante observado no atual estudo foi que a frequência de disfunção sexual detectada pelo QS-F foi maior do que a insatisfação sexual relatada pelas mulheres. Isso gera um questionamento sobre a real capacidade de avaliação da disfunção sexual a partir de tal quociente e sobre a possibilidade de questões culturais terem interferido na resposta dessas mulheres.

Esse resultado paradoxal também foi apontado em outros estudos $^{14-17}$, o que implica reflexões sobre questões diagnósticas mais precisas de disfunção sexual. Alguns estudos concordam que um problema sexual somente deve ser considerado como disfunção sexual quando causar aflição da mulher, incômodo refletido, portanto, na insatisfação com a vida sexual ${ }^{4,18}$.
Nesta pesquisa, mulheres mais jovens ( 35 a 49 anos) apresentaram $70 \%$ menos chance de terem disfunção sexual. Diversos estudos realizados com mulheres de faixa etária próxima à da atual pesquisa e utilizando outros instrumentos de avaliação da função sexual mostraram que a idade mais elevada, maior que $48 \operatorname{anos}^{15}$, igual ou maior que $50^{9}$ e igual ou maior que $55^{6,14}$, estava associada à disfunção sexual. Ainda, estudos realizados na Espanha ${ }^{19}$, na China ${ }^{20}$, na Rússia ${ }^{21}$, no Equador ${ }^{10}$ e na Turquia ${ }^{22,23}$ também confirmaram o achado de que a idade mais avançada associa-se à disfunção sexual.

Em relação ao estado civil, não houve associação de tal variável à disfunção sexual. Todavia, não há consenso na literatura. Estudos mostram que mulheres casadas apresentaram mais chances de terem disfunção sexual ${ }^{10,14,23,24}$, enquanto outro evidenciou que as divorciadas ou separadas tiveram maior frequência de disfunção ${ }^{6}$.

Quanto à escolaridade, esperar-se-ia que o menor nível educacional estivesse associado à disfunção sexual, como revelam alguns estudos ${ }^{5,20}$; no entanto, os achados não mostraram tal associação. Essa diferença poderia ser explicada pelo fato de não existir uma padronização do que é considerada baixa escolaridade.

Em relação às comorbidades, não houve associação da hipertensão arterial, depressão e diabetes melito à disfunção sexual. Resultados discordantes são encontrados na literatu$\mathrm{ra}^{9,22,25-27}$, mostrando que essas doenças estariam associadas aos piores escores de sexualidade. Tais resultados discrepantes podem ser atribuídos às diferenças metodológicas encontradas quanto ao tipo de questionário empregado (Short Personal Experiences Questionnaire e FSFI $^{20,22,25-27}$ ), à característica da população incluída (escolaridade mínima de 11 anos $^{9}$, mulheres chinesas ${ }^{20,27}$, mais de $20 \operatorname{anos}^{22}, 20$ a $60 \operatorname{anos}^{27}$, sem outras morbidades ${ }^{27}$, tipo de diabetes ${ }^{26,27}$ ) e ao tipo de análise utilizada (regressão logística ${ }^{20,27}$ ).

A osteoporose aumentou em três vezes a chance de a mulher ter disfunção sexual. Estudo de corte transversal realizado na Espanha com mulheres na pós-menopausa portadoras de osteoporose evidenciou que essa comorbidade piorou a qualidade de vida. Tal estudo observou ainda que as mulheres utilizando moduladores seletivos dos receptores de estrógeno, tipo de terapia medicamentosa para a osteoporose, apresentaram menor impacto da osteoporose na qualidade de vida, particularmente no domínio físico, avaliado pelo SF-12v2 Health Survey e no domínio sexualidade, avaliado a partir da Cervantes Scale. Esse dado confirma o achado da atual pesquisa, o que mostra a influência da osteoporose sobre a sexualidade feminina ${ }^{28}$.

A incontinência urinária elevou, em duas vezes, a chance de disfunção sexual na presente pesquisa. O resultado é semelhante ao de um estudo brasileiro com mulheres de 40 a 65 anos, no qual a incontinência urinária associou-se aos piores escores de sexualidade ${ }^{9}$, assim como se aproxima de 
um estudo latino-americano, em que problemas urinários aumentaram, em uma vez e meia, a chance de uma mulher apresentar disfunção sexual ${ }^{14}$. Essa associação pode ser um reflexo de anseios e apreensões quanto à possibilidade da perda urinária durante a relação sexual ${ }^{29}$.

O uso de terapia de reposição hormonal por seis meses ou mais não se associou à disfunção sexual na atual pesquisa. Apesar de vários estudos mostrarem melhora da função sexual com o uso da terapia de reposição hormonal ${ }^{30-33}$, uma recente revisão sistemática da Biblioteca Cochrane ${ }^{34}$, envolvendo 27 estudos com 16.393 mulheres, não apontou melhora da função sexual no subgrupo de mulheres com mais de 5 anos de finalização da menstruação, independente dos sintomas da menopausa. A revisão apresenta uma melhora de magnitude pequena ou moderada, apenas quando a terapia de reposição hormonal é utilizada em mulheres com sintomas de menopausa ou pós-menopausa nos cinco primeiros anos de amenorreia ${ }^{34}$.

Além disso, há também na literatura um estudo envolvendo 11 países da América Latina com 7.243 mulheres (40 a 59 anos) que aponta um aumento, em uma vez e meia, da chance de disfunção sexual com essa terapia ${ }^{14}$. Portanto, é possível que outros fatores possam interferir em tais resultados, como a dosagem e o tempo de uso da medicação, bem como a gravidade dos sintomas. Mulheres em uso da terapia de reposição hormonal podem estar representando os casos mais graves de sintomas na menopausa. Apesar dessa intervenção, o resultado pode não refletir diretamente na melhora da disfunção sexual. Vale ainda destacar que, na amostra do presente estudo, poucas mulheres faziam uso de tal terapia, o que pode também ter interferido nos resultados.

Quanto às cirurgias do assoalho pélvico, não houve associação da histerectomia e ooforectomia à disfunção sexual. Mais uma vez, há um dissenso na literatura pesquisada quanto a uma possível relação de causalidade entre essas variáveis ${ }^{6,14}$. Já as correções cirúrgicas do assoalho pélvico relacionaram-se à disfunção. Mulheres com histórico desse procedimento apresentaram duas vezes mais chances de terem disfunção sexual. Esse resultado é similar a uma pesquisa realizada com 33 mulheres, na qual $73 \%$ delas mostraram piora da função sexual após 6 meses de cirurgia para correção de prolapso ${ }^{35}$.

Em relação à história obstétrica, não houve associação com o parto vaginal, uso do fórceps e da episiotomia com a disfunção. No entanto, é importante destacar que os vários estudos que mostraram associação entre essas variáveis ${ }^{36-39}$ apresentaram resultados a curto prazo, diferentemente da proposta do presente estudo, o qual incluiu a faixa etária do climatério, portanto em um período de tempo mais longo do parto.

Ademais, quanto à história sexual, as mulheres que se sentiam à vontade para falar sobre sexo tiveram metade de chances de apresentarem disfunção sexual. Apesar de não terem sido encontrados estudos que avaliassem tal associação (Medline - 1966-2013, Lilacs - 1986-2013), pode-se inferir que isso se deve ao fato dessas mulheres serem mais desinibidas, o que as levam a terem um maior diálogo sobre o assunto, gerando um melhor entendimento e conhecimento sobre sua sexualidade, facilitando, dessa forma, sua busca pela satisfação sexual.

É relevante ressaltar como limitação da presente pesquisa que, por não terem sido avaliadas as possíveis disfunções sexuais dos parceiros das voluntárias, a prevalência de disfunção sexual feminina pode não ter sido fidedigna à realidade desse grupo, visto que a presença de problemas sexuais masculinos pode afetar a vida sexual das mulheres, fazendo-as acreditarem serem as disfuncionais.

Além disso, a exclusão das mulheres analfabetas, que ocorreu pelo fato de se acreditar que o auxílio de uma pessoa alfabetizada na leitura do quociente e na marcação das respostas pudesse interferir nas respostas dessas mulheres, pode ser considerada também como uma limitação deste estudo, uma vez que essa população poderia ter agregado importante contribuição aos resultados da pesquisa.

Por fim, é importante que estudos posteriores ampliem os fatores que possam estar associados à disfunção sexual em mulheres no climatério, possibilitando a construção de um modelo hierárquico com aplicação de uma regressão logística para averiguar tais fatores em uma análise multivariada.

\section{Referências}

1. Pinto Neto AM, Valadares AL, Costa-Paiva L. [Climacteric and sexuality]. Rev Bras Ginecol Obstet. 2013;35(3):93-6. Portuguese.

2. Cabral PU, Canário AC, Spyrides MH, Uchôa SA, Eleutério Júnior J, Amaral RL, et al. [Influence of menopausal symptoms on sexual function in middle-aged women]. Rev Bras Ginecol Obstet. 2012;34(7):329-34. Portuguese.

3. De Lorenzi DR, Baracat EC, Saciloto B, Padilha I Jr. Factors related to quality of life in post-menopause. Rev Assoc Med Bras. 2006;52(5):312-7.
4. Donati Sarti C, Graziottin A, Mincigrucci M, Ricci E, Chiaffarino $\mathrm{F}$, Bonaca $\mathrm{S}$, et al. Correlates of sexual functioning in Italian menopausal women. Climacteric. 2010;13(5):447-56.

5. Abdo CH, Oliveira WM Jr, Moreira ED Jr, Fittipaldi JA. Prevalence of sexual dysfunctions and correlated conditions in a sample of Brazilian women - results of the Brazilian study on sexual behavior (BSSB). Int J Impot Res. 2004; 16(2): 160-6.

6. Cabral PU, Canário AC, Spyrides MH, Uchôa SA, Eleutério J Jr, Gonçalves AK. Determinants of sexual dysfunction among middleaged women. Int J Gynaecol Obstet. 2013;120(3):271-4. 
7. De Lorenzi DR, Saciloto B. Frequência da atividade sexual em mulheres menopausadas. Rev Assoc Med Bras. 2006;52(4):256-60.

8. Araújo IA, Queiroz AB, Moura MA, Penna LH. Representações sociais da vida sexual de mulheres no climatério atendidas em serviços públicos de saúde. Texto \& Contexto Enferm. 2013;22(1):114-22.

9. Valadares AL, Pinto-Neto AM, Osis M, Conde DM, Sousa MH, Costa-Paiva L. Sexuality in Brazilian women aged 40 to 65 years with 11 years or more of formal education: associated factors. Menopause. 2008; 15(2):264-9.

10. Yánez D, Castelo-Branco C, Hidalgo LA, Chedraui PA. Sexual dysfunction and related risk factors in a cohort of middle-aged Ecuadorian women. J Obstet Gynaecol. 2006;26(7):682-6.

11. Nappi RE, Albani F, Santamaria V, Tonani S, Magri F, Martini E, et al. Hormonal and psycho-relational aspects of sexual function during menopausal transition and at early menopause. Maturitas. 2010;67(1):78-83.

12. Pongsatha S, Morakot N, Chaovisitsaree S. Sexual in menopausal women comparing perimenopausal and postmenopausal women. J Med Assoc Thai. 2012;95(12):1489-94.

13. Brasil. Ministério da Saúde. Conselho Nacional de Saúde [Internet]. Resolução n 466, de 12 de dezembro de 2012. Aprova diretrizes e normas regulamentadoras de pesquisas envolvendo seres humanos. Brasília: Ministério da Saúde; 2012 [citado 2013 Set 10]. Disponível em: http://conselho.saude.gov. $\mathrm{br} /$ resolucoes/2012/Reso466.pdf

14. Figueroa JR, Jara AD, Fuenzalida PA, del Prado AM, Flores D, Blumel JE. Prevalencia de disfunción sexual en mujeres climatéricas. Rev Méd Chil. 2009;137(3):345-50.

15. Abdo CHN, Fleury HJ. Aspectos diagnósticos e terapêuticos das disfunções sexuais femininas. Rev Psiquiatr Clin. 2006;33(3):162-7.

16. Ferenidou F, Kapoteli V, Moisidis K, Koutsogiannis I, Giakoumelos A, Hatzichristou D. Presence of a sexual problem may not affect women's satisfaction from their sexual function. J Sex Med. 2008;5(3):631-9.

17. Shifren JL, Monz BU, Russo PA, Segreti A, Johannes CB. Sexual problems and distress in United States women: prevalence and correlates. Obstet Gynecol. 2008; 112 (5):970-8.

18. Basson R, Leiblum S, Brotto L, Derogatis L, Fourcroy J, Fugl-Meyer $\mathrm{K}$, et al. Definitions of women's sexual dysfunction reconsidered: advocating expansion and revision. J Psychosom Obstet Gynaecol. $2003 ; 24(4): 221-9$.

19. Artiles Pérez V, Gutiérrez Sigler MD, Sanfélix Genovés J. [Female sexual function and related factors]. Aten Primaria. 2006;38(6):33944. Spanish

20. Lianjun P, Aixia Z, Zhong W, Feng P, Li B, Xiaona Y. Risk factors for low sexual function among urban Chinese women: a hospitalbased investigation. J Sex Med. 201 1;8(8):2299-304.

21. Kogan MI, Kalinchenko S, Avadieva NE. [Sexual dysfunction in Russia: risk factors for women]. Urologiia. 2009;(5):8-12. Russian.

22. Aslan E, Beji NK, Gungor I, Kadioglu A, Dikencik BK. Prevalence and risk factors for low sexual function in women: a study of 1,009 women in an outpatient clinic of a university hospital in Istanbul. J Sex Med. 2008;5(9):2044-52.
23. Oksuz E, Malhan S. Prevalence and risk factors for female sexual dysfunction in Turkish women. J Urol. 2006;175(2):654-8.

24. Safarinejad MR. Female sexual dysfunction in a population-based study in Iran: prevalence and associated risk factors. Int J Impot Res. 2006; 18(4):382-95.

25. De Franciscis P, Mainini G, Messalli EM, Trotta C, Luisi A, Laudando E, et al. Arterial hypertension and female sexual dysfunction in postmenopausal women. Clin Exp Obstet Gynecol. 2013;40(1):58-60.

26. Doruk H, Akbay E, Cayan S, Akbay E, Bozlu M, Acar D. Effect of diabetes mellitus on female sexual function and risk factors. Arch Androl. 2005;51(1):1-6.

27. Shi YF, Shao XY, Lou QQ, Chen YJ, Zhou HJ, Zou JY. Study on female sexual dysfunction in type 2 diabetic Chinese women. Biomed Environ Sci. 2012;25(5):557-61.

28. Palacios S, Neyro JL, Fernández de Cabo S, Chaves J, Rejas J. Impact of osteoporosis and bone fracture on health-related quality of life in postmenopausal women. Climacteric. 2014;17(1):60-70.

29. Dalpiaz O, Kerschbaumer A, Mitterberger $M$, Pinggera GM, Colleselli $D$, Bartsch $G$, et al. Female sexual dysfunction: a new urogynaecological research field. BJU Int. 2008;101(6):717-21.

30. Chedraui P, Pérez-López FR, Mezones-Holguin E, San Miguel G, Avila $\mathrm{C}$, et al. Assessing predictors of sexual function in mid-aged sexually active women. Maturitas. $2011 ; 68(4): 387-90$.

31 . Fernandes T, Costa-Paiva LH, Pinto-Neto AM. Efficacy of vaginally applied estrogen, testosterone, or polyacrylic acid on sexual function in postmenopausal women: a randomized controlled trial. J Sex Med. 2014;11(5):1262-70.

32. Jonusiene G, Zilaitiene B, Adomaitiene V, Aniuliene R, Bancroft J. Sexual function, mood and menopause symptoms in Lithuanian postmenopausal women. Climacteric. 2013;16(1):185-93.

33. Dennerstein L, Guthrie JR, Hayes RD, DeRogatis LR, Lehert P. Sexual function, dysfunction, and sexual distress in a prospective, population-based sample of mid-aged, Australian-born women. J Sex Med. 2008;5(10):2291-9.

34. Nastri CO, Lara LA, Ferriani RA, Rosa-e-Silva AC, Figueiredo JB, Martins WP. Hormone therapy for sexual function in perimenopausal and postmenopausal women. Cochrane Database Syst Rev. 2013;6:CD009672

35. Su TH, Lau HH, Huang WC, Chen SS, Lin TY, Hsieh CH, et al. Shortterm impact on female sexual function of pelvic floor reconstruction with the Prolift procedure. J Sex Med. 2009;6(1 1):3201-7.

36. Koc O, Duran B, Ozdemırcı S, Bakar $Y$, Ozengin N. Is cesarean section a real panacea to prevent pelvic organ disorders? Int Urogynecol J. 2011 ;22(9): 1135-41.

37. Rowlands IJ, Redshaw M. Mode of birth and women's psychological and physical wellbeing in the postnatal period. BMC Pregnancy Childbirth. 2012;12:138.

38. Hosseini L, Iran-Pour E, Safarinejad MR. Sexual function of primiparous women after elective cesarean section and normal vaginal delivery. Urol J. 2012;9(2):498-504.

39. Ejegård H, Ryding EL, Sjögren B. Sexuality after delivery with episiotomy: a long-term follow-up. Gynecol Obstet Invest. 2008;66(1):1-7. 